\title{
Novel Design for a Diffusive Solar Cell Window
}

\author{
Ruei-Tang Chen, Chih-Chieh Kang, Jeng-Feng Lin, Sheng-Wei Chiou, \\ Hung-Hsiang Cheng, and Chih-Wen Lai \\ Department of Electro-Optical Engineering, Southern Taiwan University of Science and Technology, Yong-Kang, Tainan 710, Taiwan
}

Correspondence should be addressed to Chih-Chieh Kang; kangc@mail.stust.edu.tw

Received 30 September 2014; Accepted 16 December 2014

Academic Editor: Giuseppe Carbone

Copyright (C) 2015 Ruei-Tang Chen et al. This is an open access article distributed under the Creative Commons Attribution License, which permits unrestricted use, distribution, and reproduction in any medium, provided the original work is properly cited.

\begin{abstract}
Building integrated photovoltaics (BIPV) are an important application of future solar energy development. The incorporation of solar cells into windows must not only maintain indoor natural lighting but also generate electrical power at the same time. In our continuing effort to improve the design of diffusion solar window, a more fundamental and efficient three-layer structureglass/EVA with $\mathrm{TiO}_{2}$ nanoparticles embedded/glass-was proposed. In this work, a well-established ASAP ray-tracing model for a diffusive solar cell window was implemented to validate the outperformance of three-layer structure over primitive five-layer structure. Optical simulations were also implemented to perform its primary design for the determination of the optimal design parameters, such as the glass thickness, the EVA thickness, and the weight concentration of $\mathrm{TiO}_{2}$ nanoparticles. Based on the simulation results, an optimal design for a three-layer diffusive solar cell window prototype was proposed. And the influence of both EVA thickness and glass thickness on the power edge-exitance (solar cell power generation efficiency) of a DSCW was thoroughly investigated.
\end{abstract}

\section{Introduction}

As one of the fast-growing segments in the solar cell industry, building integrated photovoltaics (BIPV) could become the savior of the industry with decreasing government financial subsidies globally. Among many BIPV solutions [1-5], a diffusive solar cell window (DSCW) with high transparent degrees as well as high power generation efficiency exhibits great market potential due to not only its applications in new environment-friendly zero-energy skyscrapers but also its huge potential market for mandatory energyefficient residential window replacement $[6,7]$. Primitively, a DSCW consisted of a polycarbonate (PC) diffusion plate with uniformly distributed diffusive titanium dioxide $\left(\mathrm{TiO}_{2}\right)$ nanoparticles sandwiched between two pieces of glass, and ethylene vinyl acetate (EVA) is used to laminate the glass and PC, as shown in Figure 1(a). By solely disposing thin strips of monocrystalline solar cells around the edges of the laminated glass, it generates less electricity compared to traditional BIPVs [8-10].
To enhance the competitiveness and attractiveness of this product, it is important to make it more effective and efficient by further enhancing its power generation efficiency while maintaining its natural lighting function. To achieve that objective, a novel three-layer structure of a DSCW was proposed. It comprised an EVA diffusion layer with $\mathrm{TiO}_{2}$ nanoparticles uniformly distributed which laminated two pieces of glass; its edges were surrounded by strips of monocrystalline solar cells, as shown in Figure 1(b). Instead of being only used as a hot-melt adhesive in a laminate, EVA was also applied as the medium of the diffusion layer. Accordingly, a well-established ASAP ray-tracing model for this innovative three-layer DSCW was implemented to perform its primary design. The size of a typical DSCW experimental prototype was specified in simulations. By optical simulations, its optimal design parameters, such as the thickness of both EVA and glass and the weight concentration of $\mathrm{TiO}_{2}$ nanoparticles, were determined. The construction of its experimental prototype could be followed up. The simulation 


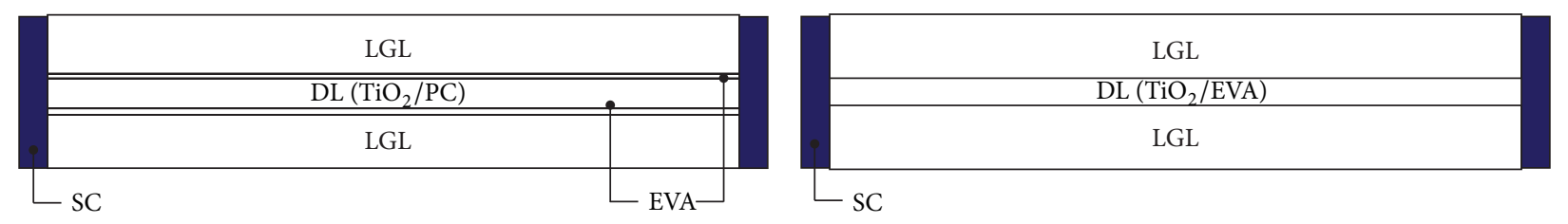

(a)

(b)

FIGURE 1: Schematics of two embodiments of a diffusive solar cell window, (a) five-layer structure and (b) three-layer structure. (DL: diffusion layer, LGL: light guide layer (glass), and SC: silicon solar cells).

results also demonstrated that the power generation efficiency of a three-layer diffusive solar cell window is indeed better than a five-layer one.

\section{Materials and Methods}

In order to validate the outperformance of our novel threelayer DSCW design over its predecessor primitive five-layer DSCW design as well as to achieve its optimal design, optical simulations were performed using ASAP, which could greatly reduce the number of costly prototyping iterations afterwards. In this study a three-layer DSCW ASAP raytracing model was developed, which was a modified optical model stemming from the previously constructed five-layer DSCW ASAP ray-tracing model [6]. The $\mathrm{TiO}_{2}$ nanoparticles applied in the model possessed an "effective average radius" of about $140 \mathrm{~nm}$, which accounted for the equivalent scattering effect of a size distribution of quasi-spherical particles. There existed two relevant versions: single-wavelength approach and multiwavelength approach. The multiwavelength approach emulated AM 1.5 global 1 sun solar illumination spectrum. The choice principle for use was based on the consideration of computation time and the necessity for color change study of transmittance, reflectance, and edgeexitance, which we defined as the proportion of amount of guided/scattered light incident onto the solar cells on its edge to total incident light into a DSCW. Surely, the edge-exitance of a DSCW was directly related to its power generation efficiency of solar cells.

By reexamining the simulation results of a five-layer DSCW in previous study, it appeared that the medium of the diffusion layer (PC) consumed too much energy of light rays. This suggested that, for further improvement in its performance, either PC had to be replaced by other medium with less loss, such as PMMA, or different kinds of structure had to emerge instead of the use of PC. Consequently, an innovative embodiment-a three-layer DSCW-was devised. In this study a set of optical simulations was performed to validate the outperformance of our new devised three-layer DSCW over a primitive five-layer one. For comparison and illustration purpose, a size of $640 \times 640 \mathrm{~mm}$ DSCW, which was adopted in our previous study on five-layer structure, was once again chosen. The cross-sectional dimensions for two different structures-both five-layer and three-layer-are shown in Figure 2. And the thickness of diffusion layer was set to be of $2 \mathrm{~mm}$ for both structures. In three-layer structure, a virtual EVA thickness of $2 \mathrm{~mm}$ was assumed for the purpose of comparison, though it might be impractical in facttoo thick for a laminate product. Due to computing time and computing capacity consideration, single-wavelength approach was adopted for this set of simulations.

In another set of optical simulations, the effect of design parameters, such as nanoparticle concentrations (ppm), diffusion layer thickness, and glass thickness, on the edgeexitance and transmittance of a three-layer DSCW was investigated for the determination of its optimal design. For future rapid prototyping/validation, the size of the DSCW was truncated and set to be of $80 \times 80 \mathrm{~mm}$ in simulations. Both single-wavelength approach and multiwavelength approach were implemented not only for comparison purpose but also for the purpose of investigating wavelength-dependent effect of scattering by multiwavelength approach. In order to clarify individual thickness effect of diffusion layer and glass on edge-exitance and transmittance over a range of nanoparticle concentrations, this set of optical simulations was further divided into two parts. In the first part, the glass thickness was given to be of $4.7 \mathrm{~mm}$. Five different thickness of EVA was chosen $(0.2 \mathrm{~mm}, 0.5 \mathrm{~mm}, 1 \mathrm{~mm}, 2 \mathrm{~mm}$, and $3 \mathrm{~mm})$ in optical simulations to examine the effect of the thickness of diffusion layer on edge-exitance and transmittance over a range of nanoparticle concentrations and to determine its optimal design as well. In the second part, the effect of glass thickness on both edge-exitance and transmittance was investigated, and the thickness of diffusion layer was determined to be of $0.2 \mathrm{~mm}$. Five different thicknesses of glass were examined: $4.7 \mathrm{~mm}, 7.9 \mathrm{~mm}, 10 \mathrm{~mm}, 15 \mathrm{~mm}$, and $20 \mathrm{~mm}$.

A last set of optical simulations was performed to evaluate and demonstrate the efficiency of a feasible optimal design of a three-layer $640 \times 640 \mathrm{~mm}$ DSCW for future prototyping. Again, both single-wavelength approach and multiwavelength approach were applied. And the performance focus was still on both the variation of edge-exitance and transmittance on nanoparticle concentrations increment. The optimal design parameters for both EVA and glass thickness could be deduced from previous two sets of optical simulations as well as the consideration of manufacturing factor: the maximum laminate thickness and the glass thickness of a window.

\section{Results and Discussion}

For a $640 \times 640 \mathrm{~mm}$ DSCW, the simulation results, as shown in Figure 3, demonstrated a consistent trend: the edge-exitance slowly increased to flat top and transmittance decreased steadily as the nanoparticle concentration (ppm) in 


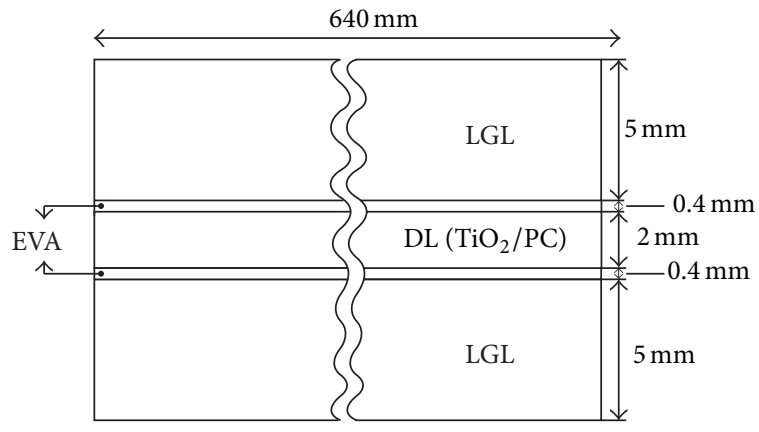

(a)

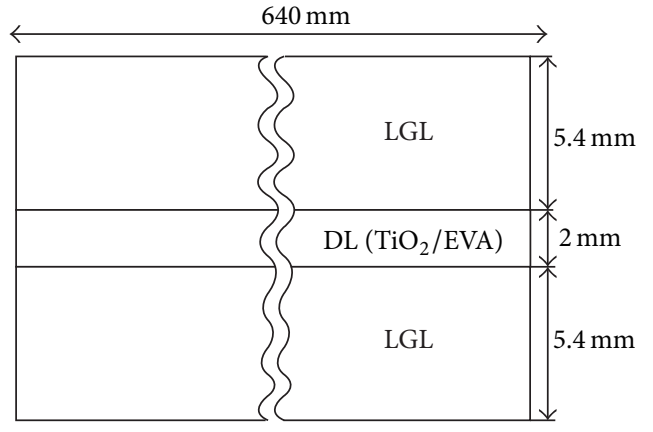

(b)

FIGURE 2: The implemented cross-sectional dimension (in proportion) for a (a) five-layer and (b) three-layer diffusive solar cell window.

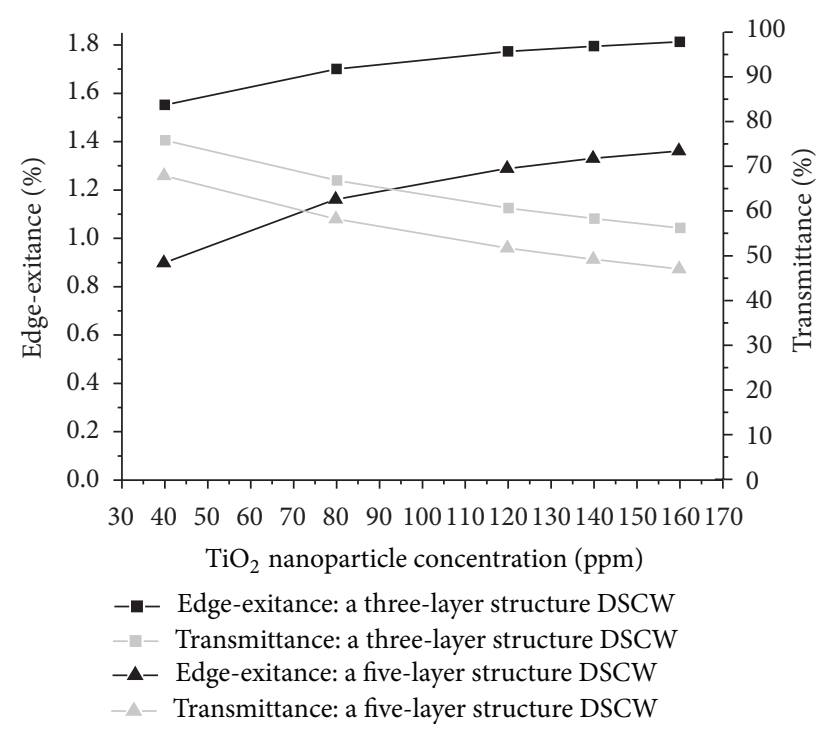

FIgURE 3: Simulation result; comparison of edge-exitance (black color) and transmittance (grey color) between a three-layer and a five-layer $640 \times 640 \mathrm{~mm}$ DSCW with different nanoparticle concentrations. Solid square (ם): three-layer structure, solid triangle $(\Delta)$ : five-layer structure.

diffusion layer increased for both three-layer and five-layer structures. In particular, further increment in nanoparticle concentrations had not any net effect on the increase of edge-exitance; on the contrary, it would only reduce transmittance after the edge-exitance had reached "saturation." In general, by comparing three-layer and five-layer structure on either edge-exitance or transmittance, it showed that threelayer structure surpassed five-layer structure. The simulation results also agreed with our hypothesis about the light loss due to the absorption of medium in a diffusion layer of a DSCW.

Due to the simplicity in structure, the task of optimal design of a three-layer DSCW had turned to be much easier. The effect of diffusion layer-EVA thickness on transmittance and edge-exitance-was determined firstly. In Figure 4, we found that the thicker the diffusion layer was, the greater the edge-exitance was and, conversely, the smaller the transmittance was. Of course, there were discrepancies in simulation results between single-wavelength approach and multiwavelength approach. In simulations, the edge-exitance (solar cell power generation efficiency) reached as high as about $12 \%$ for a $80 \times 80 \mathrm{~mm}$ DSCW with $2 \mathrm{~mm}$ thick EVA and $4.7 \mathrm{~mm}$ thick glass. With further thickness increment in EVA, there was no gain in edge-exitance. Surely, we observed once again that once the edge-exitance approached saturation, further increment in nanoparticle concentration would not help very much in edge-exitance enhancement and it could only reduce transmittance because, in practice, in most cases the thickness of the diffusion layer (EVA) is not of more than $0.5 \mathrm{~mm}$ in a laminated glass, regularly $0.1 \sim 0.2 \mathrm{~mm}$. Though unusual, it was attainable; $1 \mathrm{~mm}$ was set to be the thickness of the diffusion layer for an optimal design of a DSCW.

As for the optimal design parameter for glass thickness, we could easily observe it from simulation results depicted in Figure 5. It showed that the thicker the glass was, the larger the edge-exitance was. In simulations, the edge-exitance was as high as about $20 \%$ for a $80 \times 80 \mathrm{~mm}$ DSCW with $0.2 \mathrm{~mm}$ thick EVA and $20 \mathrm{~mm}$ thick glass. Generally, $20 \mathrm{~mm}$ thick glass could be the optimal design parameter for glass thickness in our optical simulations. However, in the consideration of glass overall cost as well as environmental factors, the thicker glass might not be the choice of consumers; the thickness of the glass for an optimal design of a DSCW was suggested to be $10 \mathrm{~mm}$ for common commercial use. After all, the design of a DSCW is a business decision; it has to consider not only power generation efficiency but natural lighting function.

In Figure 5, though the edge-exitance of a three-layer $80 \times 80 \mathrm{~mm}$ DSCW was quite high (about or above $10 \%$ in simulations), in fact its value is strongly related to its size. For an optimal design of a three-layer $640 \times 640 \mathrm{~mm}$ DSCW with $10 \mathrm{~mm}$ glass thickness and $1 \mathrm{~mm}$ EVA thickness, the simulation results showed that the edge-exitance was of less than $2.8 \%$, as shown in Figure 6. This value could be as low as one-tenths of a three-layer $80 \times 80 \mathrm{~cm}$ DSCW. This implied that the size of a DSCW could influence its edge-exitance.

As mentioned above, apart from the consideration of edge-exitance, how much transmittance is appropriate is 


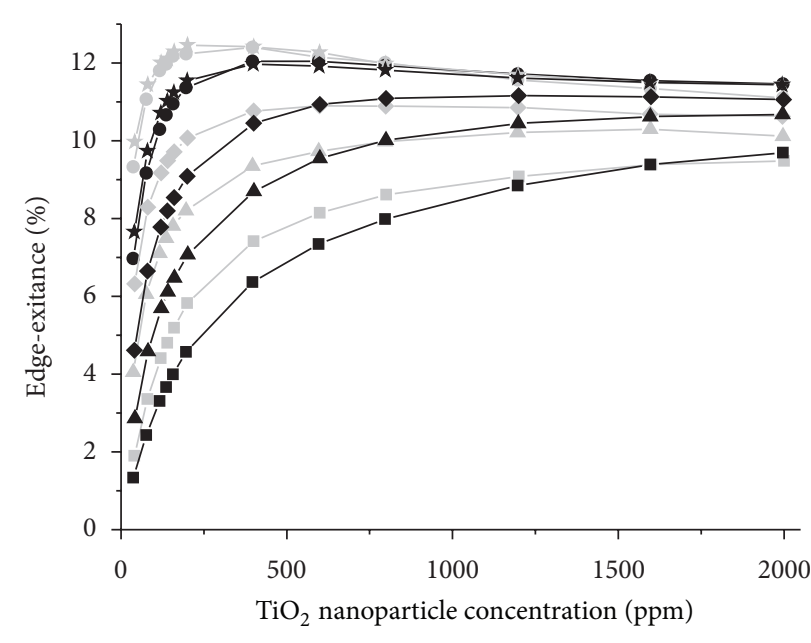

(a)

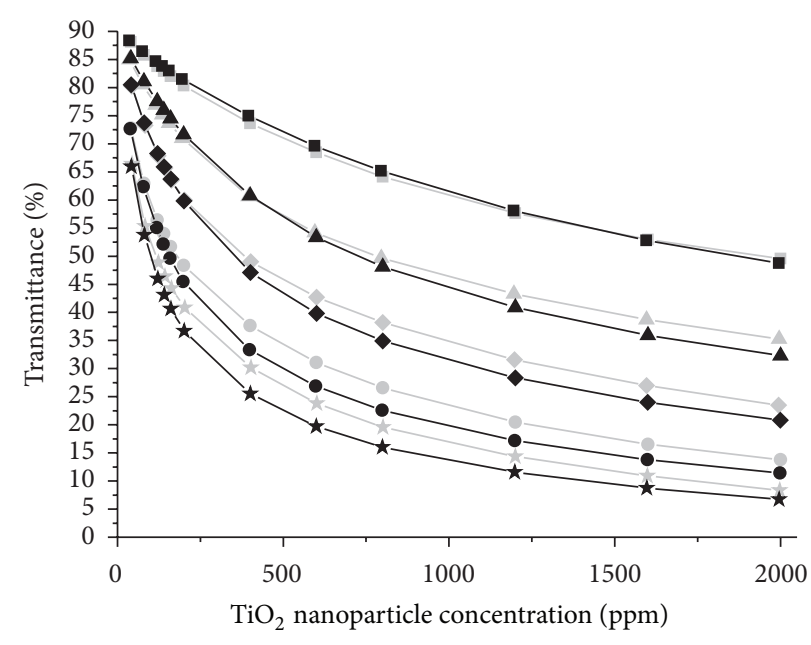

(b)

FIGURE 4: Simulation results; comparison of (a) edge-exitance and (b) transmittance of five different diffusion layer thickness of a threelayer $80 \times 80 \mathrm{~mm}$ DSCW between single-wavelength approach (grey color) and multiwavelength approach (black color) over a range of nanoparticle concentrations with $4.7 \mathrm{~mm}$ glass thickness. Solid square $(\boldsymbol{\varpi}): 0.2 \mathrm{~mm}$ thick EVA, solid triangle (A): $0.5 \mathrm{~mm}$ thick EVA, solid diamond $(\diamond)$ : $1 \mathrm{~mm}$ thick EVA, solid circle $(\bullet): 2 \mathrm{~mm}$ thick EVA, and solid star $(\star)$ : $3 \mathrm{~mm}$ thick EVA.

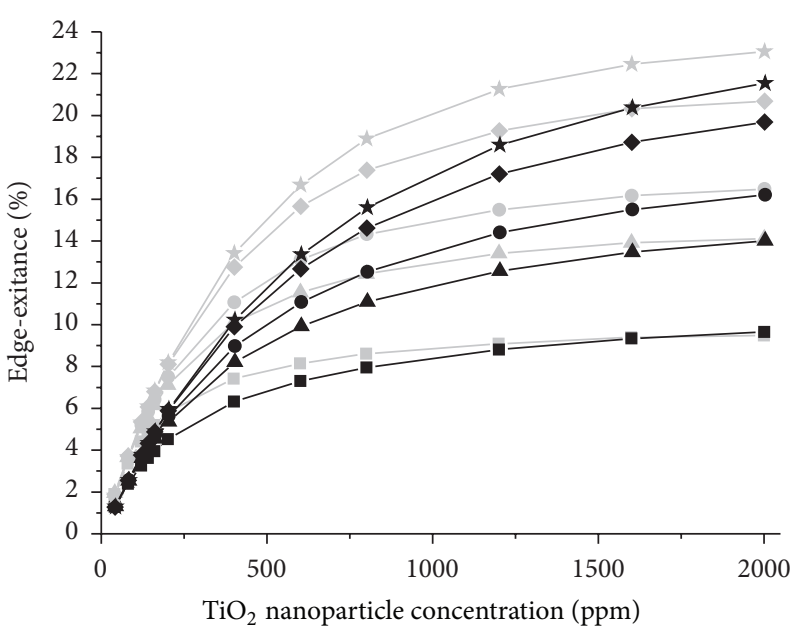

(a)

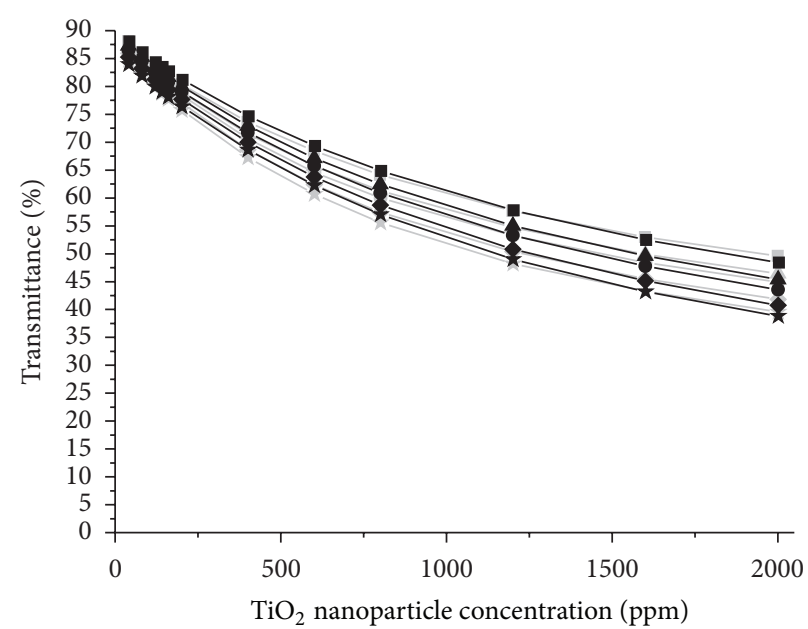

(b)

FIGURE 5: Simulation results; comparison of (a) edge-exitance and (b) transmittance of five different glass thickness of a three-layer $80 \times$ $80 \mathrm{~mm}$ DSCW between single-wavelength approach (grey color) and multiwavelength approach (black color) over a range of nanoparticle concentrations with $0.2 \mathrm{~mm}$ EVA thickness. Solid square $(\mathbf{\square}): 4.7 \mathrm{~mm}$ thick glass, solid triangle $(\mathbf{\Lambda}): 7.9 \mathrm{~mm}$ thick glass, solid circle $(\bullet): 10 \mathrm{~mm}$ thick glass, solid diamond $(\diamond)$ : $15 \mathrm{~mm}$ thick glass, and solid star $(\star)$ : $20 \mathrm{~mm}$ thick glass.

equally important in commercial use. This depends on applications and personal perception as well. The only principle established scientifically from simulation results was that once there was little enhancement in edge-exitance while increasing nanoparticle concentration, there was no need for further nanoparticle concentration increment. Otherwise meaningless loss in transmittance would become inevitable.

\section{Conclusion}

We have devised a novel three-layer diffusive solar cell window-glass/EVA with $\mathrm{TiO}_{2}$ nanoparticles embedded/ glass. Optical simulations were performed to validate its outperformance over previously developed five-layer one in terms of both transmittance and edge-exitance (solar cell power generation efficiency) by using optical simulation software-ASAP. Optical simulations for a $80 \times 80 \mathrm{~mm}$ DSCW were implemented to optimize the design of this new structure. The major design parameters were the glass thickness, EVA thickness, and nanoparticle concentration. The simulation results suggested that the thicker the glass is, the better its edge-exitance is. And it has the same effect for the thickness of diffusion layer (EVA). It also showed the edge-exitance rose to saturation and then remained almost 


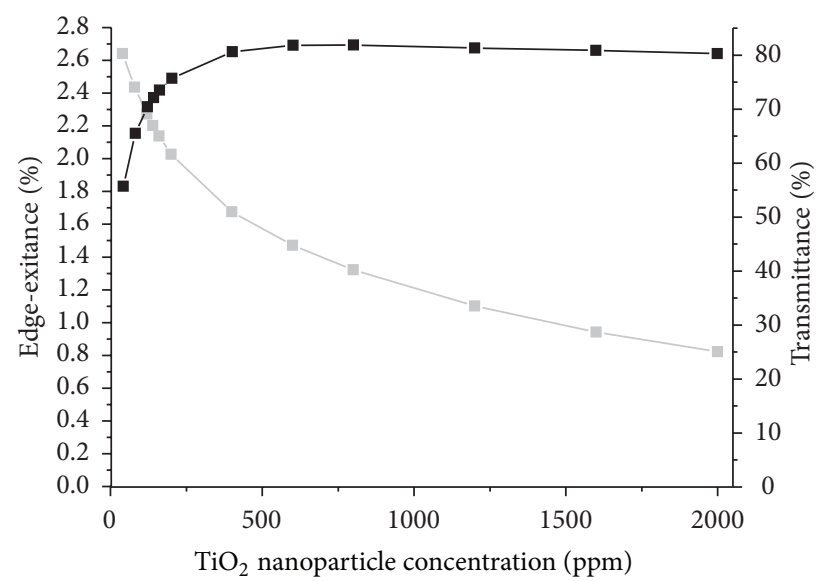

FIGURE 6: Simulation results of edge-exitance (black color) and transmittance (grey color) over a range of different nanoparticle concentrations for an optimal design of a three-layer $640 \times 640 \mathrm{~mm}$ DSCW with $10 \mathrm{~mm}$ glass thickness and $1 \mathrm{~mm}$ EVA thickness by using multiwavelength approach.

unchanged as nanoparticle concentration increases; though transmittance was decreasing accordingly. This enabled us to determine the feasible range of application for all these above-mentioned design parameters. To serve as an example for optimal design for a DSCW using optical simulation, a $640 \times 640 \mathrm{~mm}$ DSCW with $10 \mathrm{~mm}$ thick glass and $1 \mathrm{~mm}$ EVA thickness was applied. Its simulation results showed that the edge-exitance was as high as $2.8 \%$, though this value was comparatively less than the one obtained from a $80 \times$ $80 \mathrm{~cm}$ DSCW. This was due to the size effect which was deeply related to the nanoparticle concentration in EVA. The future work will involve the construction of an experimental prototype for both $80 \times 80 \mathrm{~mm}$ and $640 \times 640 \mathrm{~mm}$ DSCWs to better exploit their mechanism and validate simulation result as well.

\section{Conflict of Interests}

The authors declare that there is no conflict of interests in this study.

\section{Acknowledgments}

This work was in part supported by the Ministry of Economic Affairs (Grant no. 102-E0603) and the Ministry of Science and Technology (Grant no. NSC 102-2221-E-218-021), Taiwan.

\section{References}

[1] Y. T. Chae, J. Kim, H. Park, and B. Shin, "Building energy performance evaluation of building integrated photovoltaic (BIPV) window with semi-transparent solar cells," Applied Energy, vol. 129, pp. 217-222, 2014.

[2] M. Biancardo, K. Taira, N. Kogo et al., "Characterization of microspherical semi-transparent solar cells and modules," Solar Energy, vol. 81, no. 6, pp. 711-716, 2007.
[3] T. Miyazaki, A. Akisawa, and T. Kashiwagi, "Energy savings of office buildings by the use of semi-transparent solar cells for windows," Renewable Energy, vol. 30, no. 3, pp. 281-304, 2005.

[4] Y. Zhao and R. R. Lunt, "Transparent luminescent solar concentrators for large-area solar windows enabled by massive stokesshift nanocluster phosphors," Advanced Energy Materials, vol. 3, no. 9, pp. 1143-1148, 2013.

[5] M. J. Currie, J. K. Mapel, T. D. Heidel, S. Goffri, and M. A. Baldo, "High-efficiency organic solar concentrators for photovoltaics," Science, vol. 321, no. 5886, pp. 226-228, 2008.

[6] R.-T. Chen, C.-C. Kang, J.-F. Lin, T.-C. Lin, and C.-W. Lai, "Optimal design for the diffusion plate with nanoparticles in a diffusive solar cell window by Mie scattering simulation," International Journal of Photoenergy, vol. 2013, Article ID 481637, 5 pages, 2013.

[7] J. L. H. Chau, R.-T. Chen, G.-L. Hwang, P.-Y. Tsai, and C.-C. Lin, "Transparent solar cell window module," Solar Energy Materials and Solar Cells, vol. 94, no. 3, pp. 588-591, 2010.

[8] W. He, Y. X. Zhang, W. Sun, J. X. Hou, Q. Y. Jiang, and J. Ji, "Experimental and numerical investigation on the performance of amorphous silicon photovoltaics window in East China," Building and Environment, vol. 46, no. 2, pp. 363-369, 2011.

[9] A. Colsmann, A. Puetz, A. Bauer, J. Hanisch, E. Ahlswede, and U. Lemmer, "Efficient semi-transparent organic solar cells with good transparency color perception and rendering properties," Advanced Energy Materials, vol. 1, no. 4, pp. 599-603, 2011.

[10] K.-S. Chen, J.-F. Salinas, H.-L. Yip, L. Huo, J. Hou, and A. K.-Y. Jen, "Semi-transparent polymer solar cells with 6\% PCE, 25\% average visible transmittance and a color rendering index close to 100 for power generating window applications," Energy and Environmental Science, vol. 5, no. 11, pp. 9551-9557, 2012. 

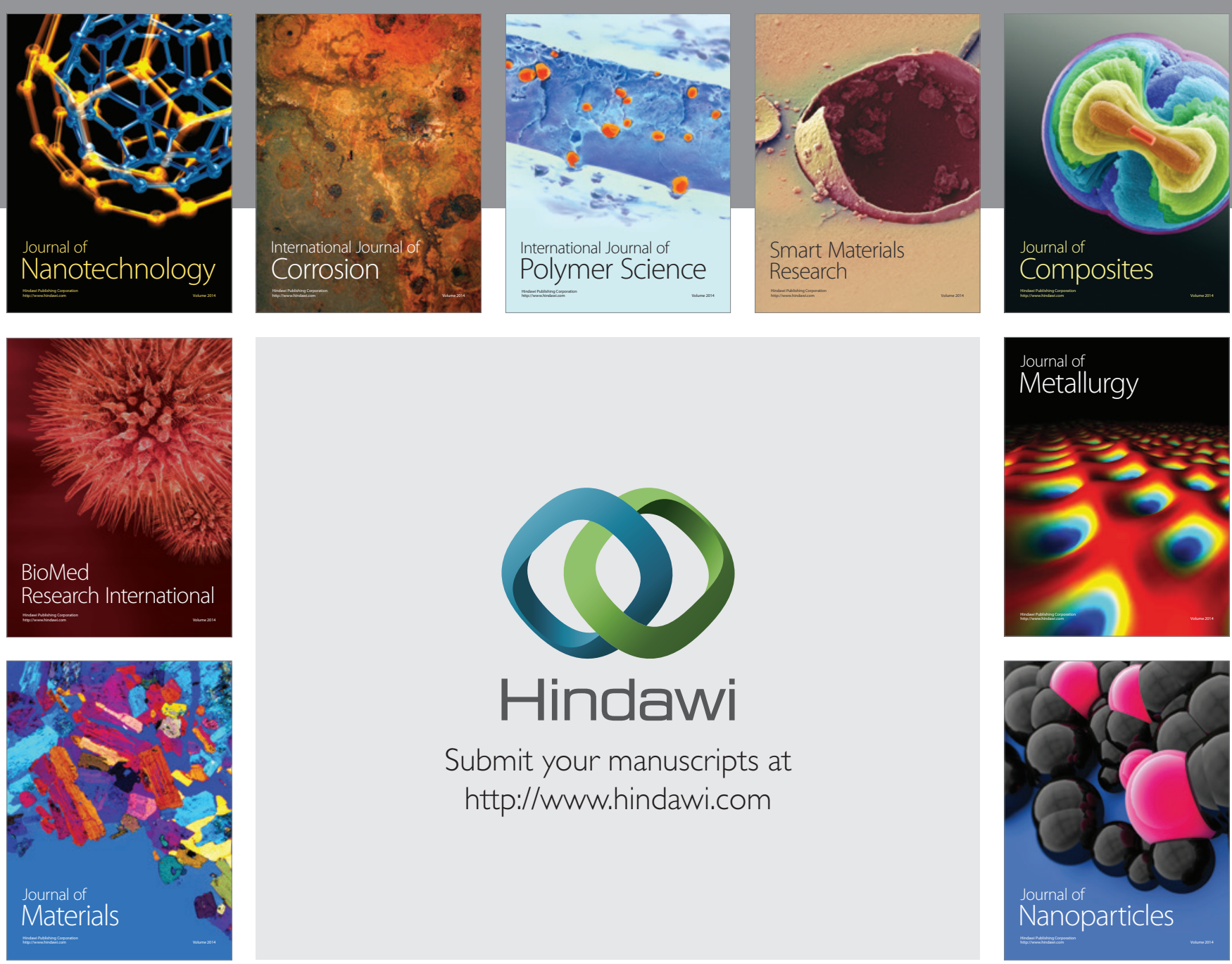

Submit your manuscripts at http://www.hindawi.com
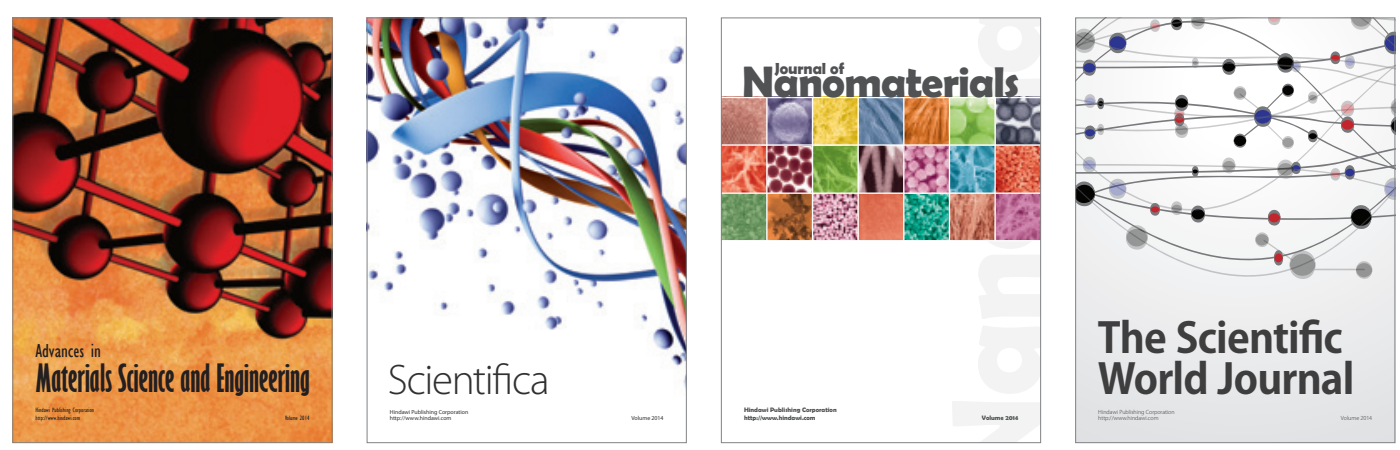

\section{The Scientific World Journal}
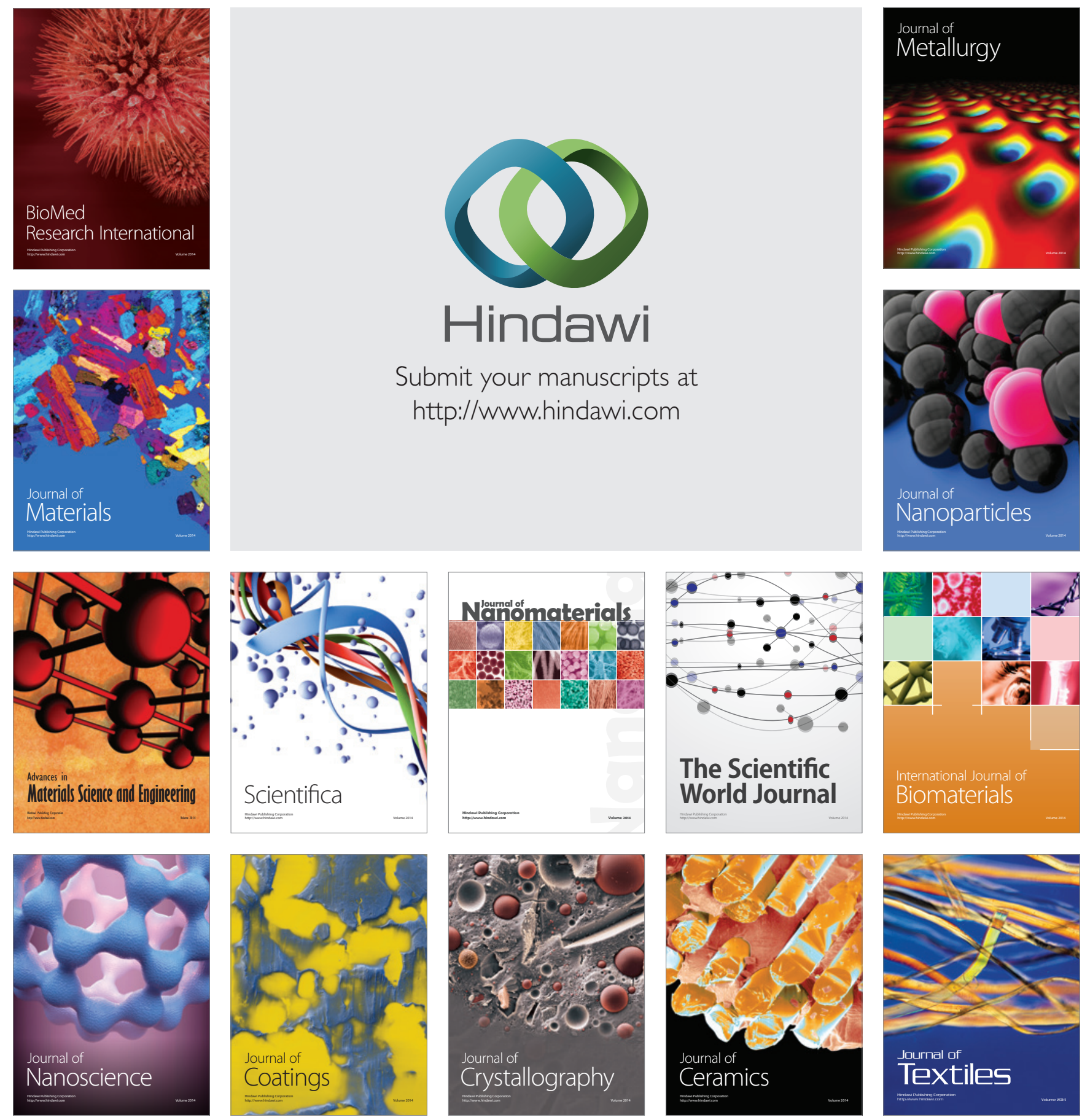